\title{
Effects of 12-week aquatic exercise on cardiorespiratory fitness, knee isokinetic function, and Western Ontario and McMaster University osteoarthritis index in patients with knee osteoarthritis women
}

\author{
Gi-Chul Ha', Jae-Ryang Yoon', Cheol-Gyu Yoo', Seol-Jung Kang ${ }^{2}$, Kwang-Jun Ko ${ }^{3, *}$ \\ 'Department of Physical Education, Korea National Sport University, Seoul, Korea \\ 2Department of Physical Education, Changwon National University, Changwon, Korea \\ ${ }^{3}$ Department of Sports Medicine, National Fitness Center, Seoul, Korea
}

The purpose of this study is to investigate the risk factors of metabolic syndrome, cardiorespiratory fitness, knee isokinetic function, and osteoarthritis index inpatients with knee osteoarthritis women. Subjects were divided into the exercise group $(n=9$, aged $60.89 \pm 5.06)$, and the control groups ( $n=8$, aged $61.25 \pm 1.91)$. Aquatic exercise was performed for 12 weeks, 3 times a week, 60 min a day. The changes of metabolic syndrome risk factors, cardiorespiratory fitness, knee isokinetic function, and WOMAC index (Western Ontario and McMaster University osteoarthritis index) were measured and analyzed at pre- and postexercise program for verifying exercise effectiveness. As a result, fasting blood glucose $(P<0.05)$, glycosylated hemoglobin $(P<0.01)$, and triglyceride $(P<0.05)$ were significantly decreased in the risk factors of metabolic syndrome. The maximum oxygen uptake in cardiorespiratory fitness was not significantly different. The left and right extensor muscles of knee isokinetic function increased significantly $(P<0.01, P<0.01$, respectively). There was no significant difference in flexor muscles. Osteoarthritis index was significantly improved in stiffness $(P<0.01)$ and physical function $(P<0.05)$. In conclusion, aquatic exercise can be regarded as an effective exercise program for managing metabolic syndrome risk factors, increasing muscle function, and improving osteoarthritis index in patients with osteoarthritis of the knee.

Keywords: Aquatic exercise, Osteoarthritis, Metabolic syndrome, Cardiorespiratory fitness, Knee isokinetic function, WOMAC index

\section{INTRODUCTION}

The incidence of musculoskeletal diseases due to population aging is increasing, and the most prevalent disease is osteoarthritis (Niu et al., 2009). Osteoarthritis, in particular, is considered a serious health problem that increases the burden of disease (Grazio, 2005). Osteoarthritis is a disease in which articular cartilage is worn or damaged, resulting in functional impairment due to repeated pain, stiffness and deformity of the joint (Arden and Nevitt, 2006; Tanaka et al., 1998). Symptoms such as pain and stiffness in osteoarthritis restrict activity and interfere with daily life (Dieppe and Lohmander, 2005). According to the WHO Sci-

entific Group on the Burden of Musculoskeletal Conditions at the Start of the New Millennium (2003), 80\% of osteoarthritis patients report mild activity limitation and $25 \%$ have difficulty performing daily activities. Thus, osteoarthritis is not a disease that leads to death, but it causes social and psychological problems caused by limitation of activity, which is a significant deterioration of quality of life.

Recent studies have shown a high prevalence of metabolic syndrome in osteoarthritis patients (Puenpatom and Victor, 2009; Yoshimura et al., 2011). Metabolic syndrome is a major risk factor for cardiovascular disease, including obesity, high blood pressure, impaired fasting glucose, dyslipidemia, and inflammatory respons-

*Corresponding author: Kwang-Jun Ko (D) https://orcid.org/0000-0002-2474-5600 Department of Sports Medicine, National Fitness Center, 424 Olympic-ro, Songpa-gu, Seoul 05540, Korea

Tel: +82-2-413-5336, Fax: +82-2-413-5533, E-mail: tigerkor80@naver.com Received: June 16, 2018 / Accepted: August 14, 2018
This is an Open Access article distributed under the terms of the Creative Commons Attribution Non-Commercial License (http://creativecommons.org/licenses/by-nc/4.0/) which permits unrestricted non-commercial use, distribution, and reproduction in any medium, provided the original work is properly cited. 
es (Libby et al., 2002). The cause of metabolic syndrome in osteoarthritis patients is the limitation of physical activity due to osteoarthritis symptoms. In addition, physical activity limitations in patients with osteoarthritis result in cardiorespiratory fitness and decreased myocardial function (Lankhorst et al., 1985). According to previous studies, the maximum oxygen uptake $\left(\mathrm{VO}_{2 \max }\right)$, which is an index of cardiorespiratory fitness in osteoarthritis patients, is only $73 \%$ of normal people (Minor et al., 1988). In a study of muscle function, quadriceps muscle strength in osteoarthritis patients was found to be weaker than normal subjects (Hurley et al., 1997; Tan et al., 1995; Wessel, 1996).

Treatment of osteoarthritis is fundamentally difficult, but it is aimed at minimizing symptoms such as pain, stiffness, and movement disorders and promoting activity (Altman, 2010). In addition, it is important to manage metabolic syndrome, improve cardiorespiratory fitness, and improve muscle function in order to lower the risk of cardiovascular disease in patients with osteoarthritis. This study was supported by a review of the literature on osteoarthritis and osteoarthritis in the elderly. However, it has been suggested that exercise with high weight-bearing may exacerbate osteoarthritis (Miyazaki et al., 2002). Therefore, it is recommended that aquatic exercise with low weight loss and low joint pressure is used in prevention or treatment of osteoarthritis (Batterham et al., 2011; Rahmann, 2010).

Aquatic exercise uses buoyancy and resistance at the same time. Because buoyancy is a force acting in the opposite direction of gravity, aquatic motion has less load than actual weight. As a result, aquatic exercise is effective in improving the pain and functional ability by lowering the mechanical burden on the knee and hip joint of osteoarthritis patients (Lim et al., 2010; Wilder and Brennan, 1993). This increase in physical activity can also affect cardiovascular disease prevention.

However, studies on cardiorespiratory fitness and muscle function, which are directly related to metabolic syndrome risk factors and activities of daily living, which are a complex of cardiovascular diseases in women with osteoarthritis of the knee, are somewhat lacking. The purpose of this study was to investigate the effect of aquatic exercise on metabolic syndrome risk factors, cardiorespiratory fitness, knee isokinetic function, and osteoarthritis index of knee osteoarthritis women.

\section{MATERIALS AND METHODS}

\section{Subjects}

The subjects of this study were middle-aged women in Seoul
Table 1. Characteristics of subjects

\begin{tabular}{lccc}
\hline Variable & Exercise group $(\mathrm{n}=9)$ & Control group $(\mathrm{n}=8)$ & $P$-value \\
\hline Age $(\mathrm{yr})$ & $60.89 \pm 5.06$ & $61.25 \pm 1.91$ & 0.846 \\
Height $(\mathrm{cm})$ & $156.89 \pm 6.99$ & $154.14 \pm 5.04$ & 0.364 \\
Weight $(\mathrm{kg})$ & $61.79 \pm 9.94$ & $58.20 \pm 10.95$ & 0.493 \\
BMI $\left(\mathrm{kg} / \mathrm{m}^{2}\right)$ & $25.18 \pm 4.31$ & $24.63 \pm 5.33$ & 0.894 \\
\hline
\end{tabular}

Values are presented as mean \pm standard deviation.

$\mathrm{BMI}$, body mass index

region who were diagnosed with osteoarthritis of knee. The subjects were selected voluntarily for participation in aquatic exercise programs, those who were diagnosed with osteoarthritis of the knee, those who received a doctor's consent to participate in the exercise program, and those who did not participate in regular exercise or other exercise programs for the past 6 months. Exercise group $(\mathrm{n}=9)$ and control group $(\mathrm{n}=8)$ were randomly selected from 17 subjects. The physical characteristics of the subjects were as shown in Table 1.

\section{Anthropometric and metabolic syndrome risk factors}

Height and weight were measured using an automatic anthropometric instrument (SH-9600A, O2run, Seoul, Korea). Body mass index (BMI) using height and weight was calculated as (body weight $[\mathrm{kg}] /$ height $[\mathrm{m}]^{2}$ ). The waist circumference was measured according to the WHO recommendation in a comfortable upright posture midway between the lowest rib and the upper extremity of the pelvic iliac crest. Body fat percentage was measured using a body fat analyzer based on bioelectrical impedance method (X-Scan, Jawon Medical, Gyeongsan, Korea). Systolic and diastolic blood pressures were measured using an automatic blood pressure monitor (FT-700R, Jawon Medical) after a stable state for more than $10 \mathrm{~min}$. Blood samples were collected from the brachial artery after fasting for at least $8 \mathrm{hr}$. After collecting the blood, centrifugation was carried out at 3,000 rpm for 15 min. Analysis of fasting blood glucose, glycosylated hemoglobin $\left(\mathrm{HbA}_{1 \mathrm{c}}\right)$, triglyceride, high-density lipoprotein cholesterol (HDL-C), and C-reactive protein (CRP) was performed with the use of a biochemical analyzer (Selecta XL, Vital Scientific, Newton, MA, USA).

\section{Cardiorespiratory test}

The cardiorespiratory fitness test was carried out using an automatic breathing gas analysis system (Q4500, Quinton, Bothell, WA, USA) to determine the $\mathrm{VO}_{2 \max }$. The protocol for testing exercise loads was the modified Balke protocol. The end of criteria for 
exercise testing was defined as rating of perceived exertion (RPE) of 17 or greater, a maximum heart rate of 5 or greater, and a respiratory exchange rate of 1.15 or greater.

\section{Knee isokinetic function test}

The knee isokinetic function of the knee was measured using the Biodex System 3 (Biodex, New York, NY, USA). After placing the subject in the measuring device chair, the axis of the knee joint and the axis of the knee joint were aligned. To prevent shaking of the body and legs during measurement, the scope of test subjects was determined after fixing the chest, abdomen, and femur with straps and fixing the legs on the resistance pads. The subject's isokinetic knee joint test protocol was performed after two exercises at an angular velocity of $60 \% \mathrm{sec}$. Before the measurement, the test method was explained to the subject.

\section{Osteoarthritis index test}

In this study, we used the Korean-WOMAC index (Bae et al., 2001) as a tool to evaluate the functional improvement of patients with osteoarthritis (WOMAC index). The Korean-WOMAC index is divided into three areas of pain, stiffness, and physical function, and consists of 24 questions. Each item is scored on a 5-point scale, and the higher the score, the worse the symptoms. The range of points in each area is $0-20$ points of pain, $0-8$ points of hardness, and 0-68 points of physical function. The WOMAC index showed higher reliability $(r=0.86)$ in assessing osteoarthritis. The Cronbach of this study was 0.95 .

\section{Aquatic exercise program}

The 12-week aquatic exercise program was conducted in the $\mathrm{K}$ indoor pool 3 times a week for $60 \mathrm{~min}$ a day, followed by a preparation exercise, a main exercise, and a healing exercise. The preparation exercise and the grooming exercise were performed for 10 min each for jumping, walking, and stretching. This exercise can be performed by swimming underwater in front of the arms, swimming back and forth in the water, raising and lowering in the water, raising the arms under the water, walking forward underwater, walking underwater, underwater squat, lifting the underwater bridge, And was performed for $40 \mathrm{~min}$. Exercise intensity was applied at 13-14 (slightly hard) level using RPE.

\section{Statistical analysis}

All data in this study were calculated using the IBM SPSS ver. 18.0 (IBM Co., Armonk, NY, USA) and presented as mean and standard deviation. Two-way analysis of variance was performed by repeated measures to verify the effect of 12 -week aquatic exercise. The significance level $(\alpha)$ of the statistical test was set at $P<0.05$.

\section{RESULTS}

\section{Changes of metabolic syndrome risk factors and CRP}

As shown in Table 2, there was no significant difference in the body weight, BMI, body fat percentage, waist circumference, systolic blood pressure, diastolic blood pressure, HDL-C, and CRP

Table 2. Change of metabolic risk factors and CRP

\begin{tabular}{|c|c|c|c|c|c|c|c|}
\hline \multirow{2}{*}{ Variable } & \multicolumn{2}{|c|}{ Exercise group } & \multicolumn{2}{|c|}{ Control group } & \multicolumn{3}{|c|}{$P$-value } \\
\hline & Pre & Post & Pre & Post & Group & Time & Interaction \\
\hline Weight (kg) & $61.79 \pm 9.94$ & $60.67 \pm 8.87$ & $58.20 \pm 10.95$ & $58.16 \pm 11.17$ & 0.548 & 0.100 & 0.122 \\
\hline $\mathrm{BMI}\left(\mathrm{kg} / \mathrm{m}^{2}\right)$ & $25.18 \pm 4.31$ & $24.76 \pm 3.96$ & $24.63 \pm 5.33$ & $24.69 \pm 5.36$ & 0.894 & 0.195 & 0.088 \\
\hline Body fat (\%) & $33.59 \pm 5.83$ & $32.63 \pm 547$ & $32.83 \pm 5.45$ & $32.49 \pm 5.41$ & 0.877 & 0.073 & 0.607 \\
\hline WC (cm) & $81.56 \pm 10.58$ & $81.50 \pm 11.10$ & $83.98 \pm 10.81$ & $84.50 \pm 10.32$ & 0.607 & 0.742 & 0.685 \\
\hline $\mathrm{SBP}(\mathrm{mmHg})$ & $116.67 \pm 17.31$ & $113.22 \pm 20.04$ & $124.88 \pm 9.72$ & $118.63 \pm 12.83$ & 0.360 & 0.075 & 0.588 \\
\hline $\mathrm{DBP}(\mathrm{mmHg})$ & $71.11 \pm 6.64$ & $70.44 \pm 7.40$ & $70.44 \pm 7.40$ & $70.44 \pm 7.40$ & 0.927 & 0.661 & 0.902 \\
\hline $\mathrm{FBG}(\mathrm{mg} / \mathrm{dL})$ & $105.33 \pm 13.01$ & $101.00 \pm 13.81$ & $114.63 \pm 20.51$ & $110.00 \pm 18.19$ & 0.258 & $0.032^{*}$ & 0.940 \\
\hline $\mathrm{HbA}_{1 \mathrm{c}}(\%)$ & $6.07 \pm 0.63$ & $5.71 \pm 0.59^{* *}$ & $6.33 \pm 1.28$ & $6.28 \pm 1.34$ & 0.409 & $0.001^{* *}$ & $0.005^{* *}$ \\
\hline $\mathrm{TG}(\mathrm{mg} / \mathrm{dL})$ & $127.44 \pm 36.97$ & $111.11 \pm 33.71$ & $118.75 \pm 31.90$ & $107.50 \pm 26.26$ & 0.702 & $0.000^{* * *}$ & 0.195 \\
\hline $\mathrm{HDL}-\mathrm{C}(\mathrm{mg} / \mathrm{dL})$ & $46.33 \pm 4.53$ & $46.78 \pm 5.72$ & $45.13 \pm 4.52$ & $46.25 \pm 3.92$ & 0.704 & 0.169 & 0.541 \\
\hline $\mathrm{CRP}$ (mg/L) & $0.17 \pm 0.16$ & $0.14 \pm 0.11$ & $0.18 \pm 0.25$ & $0.14 \pm 0.06$ & 0.975 & 0.493 & 0.905 \\
\hline
\end{tabular}

Values are presented as mean \pm standard deviation.

BMI, body mass index; WC, waist circumference; SBP, systolic blood pressure; DBP, diastolic blood pressure; FBG, fasting blood glucose; HbA $\mathrm{A}_{1 \mathrm{c}}$ glycosylated hemoglobin; TG, triglycerides; HDL-C, high-density lipoprotein cholesterol; CRP, C-reactive protein.

${ }^{*} P<0.05 .{ }^{*} P<0.01 .{ }^{* *} P<0.001$; paired $t$-test. 
Table 3. Change of cardiorespiratory fitness and knee isokinetic function

\begin{tabular}{|c|c|c|c|c|c|c|c|}
\hline \multirow{2}{*}{ Variable } & \multicolumn{2}{|c|}{ Exercise group } & \multicolumn{2}{|c|}{ Control group } & \multicolumn{3}{|c|}{$P$-value } \\
\hline & Pre & Post & Pre & Post & Group & Time & Interaction \\
\hline $\mathrm{VO}_{2 \max }(\mathrm{mL} / \mathrm{kg} / \mathrm{min})$ & $24.38 \pm 6.29$ & $27.90 \pm 2.84$ & $25.46 \pm 7.41$ & $25.19 \pm 4.44$ & 0.733 & 0.219 & 0.154 \\
\hline Rt Knee EX TQ/BW (Nm) & $126.97 \pm 26.74$ & $148.36 \pm 25.50^{* *}$ & $130.75 \pm 18.32$ & $133.39 \pm 18.00$ & 0.608 & $0.001^{* *}$ & $0.005^{* * *}$ \\
\hline Lt Knee EX TQ/BW (Nm) & $137.12 \pm 40.28$ & $151.71 \pm 43.24^{* *}$ & $128.78 \pm 27.91$ & $130.88 \pm 30.16$ & 0.420 & $0.000^{* * *}$ & $0.004^{* *}$ \\
\hline Rt Knee FX TQ/BW (Nm) & $55.11 \pm 27.12$ & $57.78 \pm 27.74$ & $51.75 \pm 18.19$ & $52.13 \pm 16.57$ & 0.687 & 0.596 & 0.689 \\
\hline Lt Knee FX TQ/BW (Nm) & $58.88 \pm 26.61$ & $60.78 \pm 22.10$ & $54.86 \pm 18.55$ & $55.63 \pm 21.62$ & 0.671 & 0.651 & 0.846 \\
\hline
\end{tabular}

Values are presented as mean \pm standard deviation.

$\mathrm{VO}_{2 m a x}$, maximum oxygen uptake; Rt Knee EX TQ/BW, right knee-extensor peak torque/body weight; Lt Knee EX TQ/BW, left knee-extensor peak torque/body weight; Rt Knee FX TQ/BW, right knee-flexion peak torque/body weight; Lt Knee FX TQ/BW, left knee-flexion peak torque/body weight.

${ }^{*} P<0.05 .{ }^{*} P<0.01 .{ }^{* *} P<0.001$; paired $t$-test.

Table 4. Change of WOMAC index

\begin{tabular}{|c|c|c|c|c|c|c|c|}
\hline \multirow{2}{*}{ Variable } & \multicolumn{2}{|c|}{ Exercise group } & \multicolumn{2}{|c|}{ Control group } & \multicolumn{3}{|c|}{$P$-value } \\
\hline & Pre & Post & Pre & Post & Group & Time & Interaction \\
\hline Pain (score) & $10.89 \pm 3.62$ & $9.22 \pm 3.60$ & $12.50 \pm 6.32$ & $11.50 \pm 4.63$ & 0.371 & 0.101 & 0.668 \\
\hline Stiffness (score) & $4.56 \pm 1.81$ & $3.56 \pm 1.59^{*}$ & $4.38 \pm 1.85$ & $5.38 \pm 2.83^{*}$ & 0.393 & 1.000 & $0.013^{*}$ \\
\hline Physical function (score) & $34.78 \pm 13.63$ & $29.33 \pm 12.65^{* *}$ & $34.88 \pm 11.59$ & $33.63 \pm 11.11$ & 0.716 & $0.003^{* *}$ & $0.003^{* *}$ \\
\hline Total (score) & $50.22 \pm 17.74$ & $42.11 \pm 16.14^{* *}$ & $51.75 \pm 19.23$ & $50.38 \pm 18.06$ & 0.573 & $0.007^{* *}$ & $0.042^{*}$ \\
\hline
\end{tabular}

Values are presented as mean \pm standard deviation.

WOMAC index, Western Ontario \& McMaster Universities Osteoarthritis index.

${ }^{*} P<0.05 .{ }^{* *} P<0.01$; paired $t$-test.

between the two groups according to the treatment. Fasting blood glucose significantly decreased after exercise in both exercise and control groups $(P<0.05)$. However, the interaction effect did not appear. $\mathrm{HbA}_{1 \mathrm{c}}$ significantly decreased after exercise in the exercise group compared to the control group $(P<0.01)$, but there was no significant difference in the control group compared to the control group. Interaction effects also appeared $(P<0.01)$. Triglyceride was decreased after exercise in both exercise and control groups $(P<0.01)$, but no interaction effect was observed.

\section{Changes of cardiorespiratory fitness and knee isokinetic muscle function}

As shown in Table 3, there was no significant difference in maximal oxygen uptake as a cardiovascular fitness index according to treatment. The left extensor muscle was significantly increased after exercise in the exercise group $(P<0.01)$. However, there was no significant difference in the control group. Interaction effects also appeared $(P<0.01)$. In the exercise group, the right extrinsic muscle increased significantly $(P<0.01)$. However, there was no significant difference in the control group. Interaction effects also appe-ared $(P<0.01)$. There was no significant difference between the two groups according to the treatment. No interaction effect was seen.

\section{Changes of osteoarthritis index (WOMAC index)}

As shown in Table 4, osteoarthritis index did not show any significant difference between the two groups according to the treatment. Stiffness decreased significantly after exercise in the exercise group $(P<0.05)$. However, the control group was significantly increased $(P<0.05)$. Interaction effects also appeared $(P<0.01)$. Physical function was significantly decreased after exercise $(P<0.01)$ in the exercise group compared to the control group. However, there was no significant difference in the control group. Interaction effects also appeared $(P<0.01)$. The overall score of osteoarthritis index decreased significantly after exercise in the exercise group $(P<0.01)$. However, there was no significant difference in the control group. Interaction effects also appeared $(P<0.05)$.

\section{DISCUSSION}

This study was conducted to investigate the risk factors of metabolic syndrome, cardiorespiratory fitness, knee isokinetic function and osteoarthritis index in patients with knee osteoarthritis women. To summarize the results of this study, 12-week aquatic exercise in women with osteoarthritis was found to be effective in improving fasting blood glucose, $\mathrm{HbA}_{\mathrm{lc}}$, triglyceride reduction, knee extensor promotion, and osteoarthritis index. 
Metabolic syndrome is characterized by abdominal obesity, impaired fasting glucose, hypertension, dyslipidemia, and is known to increase the risk of cardiovascular disease and type 2 diabetes due to atherosclerosis (Lakka et al., 2002; Malik et al., 2004). Wang et al. (2016) studies have shown a link between osteoarthritis and metabolic syndrome. The results of this study showed that the $\mathrm{HbA}_{\mathrm{lc}}$ was decreased in the exercise group, but the control group was not changed, reflecting the fasting blood glucose level and the 2-3 month average blood glucose control status in the metabolic syndrome indicator. The triglyceride was significantly decreased before and after treatment. On the other hand, there was no significant difference in obesity index, systolic and diastolic blood pressure, and HDL-C. Although not osteoarthritis patients, Kasprzak et al. (2014) reported that aquatic exercise is partially effective in altering the metabolic syndrome in obese middle-aged and elderly women. More future clinical studies are needed for the aquatic and metabolic syndrome indicators in patients with osteoarthritis later. In addition, it is necessary to examine various experimental variables such as dietary intake and stress, which affect the index of metabolic syndrome when applying aquatic exercise to osteoarthritis patients.

Osteoarthritis is a noninflammatory arthritis, but it is known that inflammation-related changes occur when cartilage destruction occurs (Myers et al., 1992). CRP reflects biological markers of inflammatory response, metabolic syndrome, and cardiovascular disease risk (Cesari et al., 2003; Ridker, 2001; Wilson et al., 2006). Exercise has been shown to have anti-inflammatory effects (Panagiotakos et al., 2005; Pedersen, 2006). The results of this study showed no significant difference in CRP. The CRP of osteoarthritis patients in this study is in the normal range. Therefore, it is considered that the change is not caused by the aquatic exercise. Pain, joint function limitation, and muscle weakness, which are the main symptoms of osteoarthritis, complicate restraint and discomfort in life, such as walking and stair climbing (Creamer et al., 2000). As a result, a decrease in physical activity lowers cardiorespiratory fitness and muscle function. Particularly low cardiorespiratory fitness increases the risk of cardiovascular disease (Carnethon et al., 2003). As a result of this study, $\mathrm{VO}_{2 \max }$, an index of cardiorespiratory fitness, was increased but no significant difference was observed. On the other hand, in the exercise group, the extensor muscles of knee isokinetic muscular function were significantly increased, but the control group did not change. Wang et al. (2007) also found that aquatic exercise was effective in increasing strength in osteoarthritis patients. In this way, aquatic exercise can be effectively applied to increase the strength of the knee joint while reducing the mechanical burden on the joints having a high weight. However, there was no difference in flexor muscles in this study. Baratta et al. (1988) reported that cooperative activity between the main and antagonistic muscles of the knee plays an essential role in maintaining joint stability and supporting ligaments. Hortobágyi et al. (2005) also suggest that it is important for patients with osteoarthritis to enhance the balance between quadriceps muscle strength and hamstring muscle strength. This suggests that additional programs are needed to improve the function of the flexor muscles as well as the extensor muscles of the knee during aquatic exercise.

Pain and stiffness in osteoarthritis patients are the main limiting factor in everyday life (Jakobsson and Hallberg, 2006). In this study, the osteoarthritis index (WOMAC index) was used to test the efficacy of aquatic exercise in osteoarthritis women. Osteoarthritis index is useful in clinical evaluation of pain, stiffness and physical function status in osteoarthritis patients. A meta-analysis of exercise programs and pain assessment tools in osteoarthritis patients showed that exercise was effective in pain relief (Lu et al., 2015). The results of this study also showed that the osteoarthritis index of the exercise group improved joint stiffness and physical function, but the control group remained unchanged. Previous studies have also shown that aquatic exercise in osteoarthritis patients is effective in improving osteoarthritis index (Foley et al., 2003; Lin et al., 2004; Silva et al., 2008). Because osteoarthritis dysfunction is related to weakness of the quadriceps muscle, improvement in muscle function is important (O’Reilly et al., 1998). Therefore, improvement of osteoarthritis index can be regarded as a result of reducing mechanical load on the knee joint due to extensor muscle strength enhancement shown in this study.

In conclusion, aquatic exercise can be regarded as an effective exercise program for the management of metabolic syndrome, improvement of muscle function, and improvement of osteoarthritis index in osteoarthritis women.

\section{CONFLICT OF INTERST}

No potential conflict of interest to this article was reported.

\section{REFERENCES}

Altman RD. Early management of osteoarthritis. Am J Manag Care 2010; 16 Suppl Management:S41-47.

Arden N, Nevitt MC. Osteoarthritis: epidemiology. Best Pract Res Clin Rheumatol 2006;20:3-25. 
Bae SC, Lee HS, Yun HR, Kim TH, Yoo DH, Kim SY. Cross-cultural adaptation and validation of Korean Western Ontario and McMaster Universities (WOMAC) and Lequesne osteoarthritis indices for clinical research. Osteoarthritis Cartilage 2001;9:746-750.

Baratta R, Solomonow M, Zhou BH, Letson D, Chuinard R, D'Ambrosia R. Muscular coactivation. The role of the antagonist musculature in maintaining knee stability. Am J Sports Med 1988;16:113-122.

Batterham SI, Heywood S, Keating JL. Systematic review and meta-analysis comparing land and aquatic exercise for people with hip or knee arthritis on function, mobility and other health outcomes. BMC Musculoskelet Disord 2011;12:123.

Carnethon MR, Gidding SS, Nehgme R, Sidney S, Jacobs DR Jr, Liu K. Cardiorespiratory fitness in young adulthood and the development of cardiovascular disease risk factors. JAMA 2003;290:3092-3100.

Cesari M, Penninx BW, Newman AB, Kritchevsky SB, Nicklas BJ, SuttonTyrrell K, Tracy RP, Rubin SM, Harris TB, Pahor M. Inflammatory markers and cardiovascular disease (The Health, Aging and Body Composition [Health ABC] Study). Am J Cardiol 2003;92:522-528.

Creamer P, Lethbridge-Cejku M, Hochberg MC. Factors associated with functional impairment in symptomatic knee osteoarthritis. Rheumatology (Oxford) 2000;39:490-496.

Dieppe PA, Lohmander LS. Pathogenesis and management of pain in osteoarthritis. Lancet 2005;365:965-973.

Grazio S. Osteoarthritis--epidemiology, economics and quality of life. Reumatizam 2005;52:21-29.

Foley A, Halbert J, Hewitt T, Crotty M. Does hydrotherapy improve strength and physical function in patients with osteoarthritis--a randomised controlled trial comparing a gym based and a hydrotherapy based strengthening programme. Ann Rheum Dis 2003;62:1162-1167.

Hortobágyi T, Westerkamp L, Beam S, Moody J, Garry J, Holbert D, DeVita P. Altered hamstring-quadriceps muscle balance in patients with knee osteoarthritis. Clin Biomech (Bristol, Avon) 2005;20:97-104.

Hurley MV, Scott DL, Rees J, Newham DJ. Sensorimotor changes and functional performance in patients with knee osteoarthritis. Ann Rheum Dis 1997;56:641-648.

Jakobsson U, Hallberg IR. Quality of life among older adults with osteoarthritis: an explorative study. J Gerontol Nurs 2006;32:51-60.

Kasprzak Z, Pilaczyńska-Szcześniak L. Effects of regular physical exercises in the water on the metabolic profile of women with abdominal obesity. J Hum Kinet 2014;41:71-79.

Lakka HM, Laaksonen DE, Lakka TA, Niskanen LK, Kumpusalo E, Tuomilehto J, Salonen JT. The metabolic syndrome and total and cardiovascular disease mortality in middle-aged men. JAMA 2002;288:27092716.

Lankhorst GJ, Van de Stadt RJ, Van der Korst JK. The relationships of functional capacity, pain, and isometric and isokinetic torque in osteoarthrosis of the knee. Scand J Rehabil Med 1985;17:167-172.

Libby P, Ridker PM, Maseri A. Inflammation and atherosclerosis. Circulation 2002;105:1135-1143.

Lim JY, Tchai E, Jang SN. Effectiveness of aquatic exercise for obese patients with knee osteoarthritis: a randomized controlled trial. PM R 2010;2: 723-731.

Lin SY, Davey RC, Cochrane T. Community rehabilitation for older adults with osteoarthritis of the lower limb: a controlled clinical trial. Clin Rehabil 2004;18:92-101.

Lu M, Su Y, Zhang Y, Zhang Z, Wang W, He Z, Liu F, Li Y, Liu C, Wang Y, Sheng L, Zhan Z, Wang $X$, Zheng N. Effectiveness of aquatic exercise for treatment of knee osteoarthritis: systematic review and meta-analysis. Z Rheumatol 2015;74:543-552.

Malik S, Wong ND, Franklin SS, Kamath TV, L'Italien GJ, Pio JR, Williams GR. Impact of the metabolic syndrome on mortality from coronary heart disease, cardiovascular disease, and all causes in United States adults. Circulation 2004;110:1245-1250.

Minor MA, Hewett JE, Webel RR, Dreisinger TE, Kay DR. Exercise tolerance and disease related measures in patients with rheumatoid arthritis and osteoarthritis. J Rheumatol 1988;15:905-911.

Miyazaki T, Wada M, Kawahara H, Sato M, Baba H, Shimada S. Dynamic load at baseline can predict radiographic disease progression in medial compartment knee osteoarthritis. Ann Rheum Dis 2002;61:617-622.

Myers SL, Flusser D, Brandt KD, Heck DA. Prevalence of cartilage shards in synovium and their association with synovitis in patients with early and endstage osteoarthritis. J Rheumatol 1992;19:1247-1251.

Niu J, Zhang YQ, Torner J, Nevitt M, Lewis CE, Aliabadi P, Sack B, Clancy M, Sharma L, Felson DT. Is obesity a risk factor for progressive radiographic knee osteoarthritis? Arthritis Rheum 2009;61:329-335.

O'Reilly SC, Jones A, Muir KR, Doherty M. Quadriceps weakness in knee osteoarthritis: the effect on pain and disability. Ann Rheum Dis 1998; 57:588-594.

Panagiotakos DB, Pitsavos C, Chrysohoou C, Kavouras S, Stefanadis C; ATTICA Study. The associations between leisure-time physical activity and inflammatory and coagulation markers related to cardiovascular disease: the ATTICA Study. Prev Med 2005;40:432-437.

Pedersen BK. The anti-inflammatory effect of exercise: its role in diabetes and cardiovascular disease control. Essays Biochem 2006;42:105-117.

Puenpatom RA, Victor TW. Increased prevalence of metabolic syndrome in individuals with osteoarthritis: an analysis of NHANES III data. Postgrad Med 2009;121:9-20.

Rahmann AE. Exercise for people with hip or knee osteoarthritis: a comparison of land-based and aquatic interventions. Open Access J Sports Med 2010;1:123-135. 
Ridker PM. High-sensitivity C-reactive protein: potential adjunct for global risk assessment in the primary prevention of cardiovascular disease. Circulation 2001;103:1813-1818.

Silva LE, Valim V, Pessanha AP, Oliveira LM, Myamoto S, Jones A, Natour J. Hydrotherapy versus conventional land-based exercise for the management of patients with osteoarthritis of the knee: a randomized clinical trial. Phys Ther 2008;88:12-21.

Tan J, Balci N, Sepici V, Gener FA. Isokinetic and isometric strength in osteoarthrosis of the knee. A comparative study with healthy women. Am J Phys Med Rehabil 1995;74:364-369.

Tanaka S, Hamanishi C, Kikuchi H, Fukuda K. Factors related to degradation of articular cartilage in osteoarthritis: a review. Semin Arthritis Rheum 1998;27:392-399.

Yoshimura N, Muraki S, Oka H, Kawaguchi H, Nakamura K, Akune T. Association of knee osteoarthritis with the accumulation of metabolic risk factors such as overweight, hypertension, dyslipidemia, and impaired glucose tolerance in Japanese men and women: the ROAD study. J Rheumatol 2011;38:921-930.
Wang H, Cheng Y, Shao D, Chen J, Sang Y, Gui T, Luo S, Li J, Chen C, Ye Y, Yang Y, Li Y, Zha Z. Metabolic syndrome increases the risk for knee osteoarthritis: a meta-analysis. Evid Based Complement Alternat Med 2016;2016:7242478.

Wang TJ, Belza B, Elaine Thompson F, Whitney JD, Bennett K. Effects of aquatic exercise on flexibility, strength and aerobic fitness in adults with osteoarthritis of the hip or knee. J Adv Nurs 2007;57:141-152.

Wessel J. Isometric strength measurements of knee extensors in women with osteoarthritis of the knee. J Rheumatol 1996;23:328-331.

WHO Scientific Group on the Burden of Musculoskeletal Conditions at the Start of the New Millennium. The burden of musculoskeletal conditions at the start of the new millennium. World Health Organ Tech Rep Ser 2003;919:i-x, 1-218, back cover.

Wilder RP, Brennan DK. Physiological responses to deep water running in athletes. Sports Med 1993;16:374-380.

Wilson AM, Ryan MC, Boyle AJ. The novel role of C-reactive protein in cardiovascular disease: risk marker or pathogen. Int J Cardiol 2006; 106:291-297. 